\title{
Um Método para Determinar a Complexidade de uma Microtask em Ambientes Crowdsourcing
}

\section{Title: A Method to Determine the Complexity of a Microtask in Crowdsourcing Environments}

\author{
William Simão de Deus' ${ }^{1}$, José Augusto Fabri', Alexandre L'Erario' \\ Programa de Pós-Graduação em Informática - Universidade Tecnológica Federal do \\ Paraná (UTFPR) \\ Cornélio Procópio, Paraná - Brasil \\ williamsimao@outlook.com, \{fabri, aleario\}@utfpr.edu.br
}

\begin{abstract}
Crowdsourcing (CS) software development employs the outsourcing of design, development and testing tasks, known as microtasks, to an anonymous group of participants. The use of microtaks in CS environments has been promoting the reduction of costs and diminution of the time of a project, which remits its importance. Despite this, the literature still does not concentrate an effective way on how to determine its difficulty of execution. Thus, the objective of this work is to present a method capable of evaluating the complexity that a microtask possesses. For this a method of determining complexity based on the characteristics of a microtask, was generated. Such a method was evaluated via a case study on a CS software development platform. The results showed that the method was efficient achieving an index of close to $90 \%$ of assertiveness in classifying microtasks as simple or complex in the analyzed sample. In addition, the method also showed that the simpler microtasks achieved a higher index of registration and submission than the complex microtasks.
\end{abstract}

Keywords. Microtasks; Complexity; Crowdsourcing Software Development; Software Complexity.

Resumo. O desenvolvimento de software crowdsourcing (CS) emprega a terceirização das tarefas de design, desenvolvimento e teste, conhecidas como microtasks, para um grupo anônimo de participantes. $O$ uso das microtaks em ambientes $C S$ vem promovendo a diminuição de custos e redução de tempo de um projeto, o que remete sua importância. Apesar disso, a literatura ainda não concentra uma maneira eficaz sobre como determinar a sua dificuldade de execução. Assim, o objetivo deste trabalho é apresentar um método capaz de avaliar a complexidade que uma microtask possui. Para isso foi gerado um método de determinação de complexidade baseado nas próprias características de uma microtask. Tal método foi avaliado via estudo de caso em uma plataforma de desenvolvimento de software CS. Os resultados mostraram que o método foi eficiente alcançando um índice próximo de $90 \%$ de assertividade em classificar microtasks como simples ou complexas na amostra analisada. Além disso, o método também evidenciou que as microtasks mais simples

Cite as: $\quad$ Deus, W. S., Fabri, J. A. \& L'Erario, A. (2019). Um Método para Determinar a Complexidade de uma Microtask em Ambientes Crowdsourcing. iSys: Revista Brasileira de Sistemas de Informação (Brazilian Journal of Information Systems), 11(4), 5-30. 
alcançaram maior índice de registro e submissão em relação às microtasks complexas.

Palavras-Chave. Microtasks; Complexidade; Desenvolvimento de Software Crowdsourcing; Complexidade de Software.

\section{Introdução}

O crowdsourcing (CS) é um modelo de negócios que utiliza uma chamada aberta para atração de colaboradores anônimos no desenvolvimento de atividades ou na solução de problemas [Howe 2006b]. Sendo assim, o CS representa o ato de uma pessoa ou organização terceirizar uma porção de trabalho para um grupo de colaboradores anônimos, especialistas ou entusiastas, interessados na execução de uma atividade [Mao et al. 2017].

O CS é um modelo emergente no setor produtivo de software. Isso se deve ao fato de empresas de grande porte buscarem soluções diversificadas, rapidez e paralelização de atividades em plataformas online que suportam o desenvolvimento de software CS [TopCoder 2017, de Deus et al. 2016]. Dessa forma, com o mercado aquecido, diversas plataformas são criadas para suprir a crescente demanda industrial [Dwarakanath et al. 2015].

O desenvolvimento de software CS suportado nessas plataformas é baseado em atividades de curta duração para programação, teste ou design de um projeto de software [LaToza et al.2014]. Essas atividades são conhecidas como microtasks por representarem pequenas porções de trabalho, reduzirem custos e possuírem curtos prazos de execução. Porém, a literatura ainda é incipiente e possui diversas lacunas relacionadas ao modo de mensurar a complexidade de uma microtask.

Em um mapeamento sistemático realizado por [de Deus et al. 2017] os autores revisaram a literatura e demonstraram que as a complexidade das microtasks é um dos principais fatores que torna complexo o gerenciamento de projetos CS. Além disso, o mapeamento identificou uma carência de estudos sobre métodos abordando o assunto de complexidade de execução. Os estudos revelados pelo mapeamento foram desenvolvidos apenas para suportar apenas projetos CS complexos, ou seja, projetos que possuem várias microtasks ou muitos participantes [Xiao e Paik 2014, Kittur et al. 2011].

Ademais, os mecanismos e métricas tradicionais adotados para mensurar a complexidade de atividades de software nos modelos de desenvolvimento similares não podem ser adotados diretamente em projetos CS. Segundo [Naik 2016] o CS é um modelo de desenvolvimento único que apresenta variáveis diferentes e mais complexas que outros modelos de desenvolvimento devido aos participantes anônimos e dispersos, atomicidade das microtasks e remuneração entre os pares. Desse modo, as métricas existentes para aferir a complexidade de atividades ou projetos de software apresentam falhas durante sua aplicação nas microtasks executadas em ambientes CS.

Nesse sentido é importante destacar o motivo pelo qual as métricas disponíveis (como Mood, Chidamber-Kemerer (CK), Halstead, complexidade Ciclomática (CC), Mean Time Between Failures (MTBF) e suas variações) não podem ser aplicadas nas microtasks. As métricas Mood e CK descrevem o uso de códigos de software baseado no paradigma orientado a objetos. Desse modo, ambas as métricas utilizam um mecanismo de encapsulamento, herança, polimorfismo e troca de mensagens para calcular a 
complexidade de um sistema ou atividade de software [Chandra et al. 2017]. Entretanto, de acordo com [Mao et al. 2017], o CS utiliza microtasks voltadas a etapa de design, mockups e protótipos e as métricas Mood e CK não conseguem aferir a complexidade delas.

Esse problema é similar ao encontrado na métrica Halstead que consegue aferir a complexidade algorítmica, descartando os demais tipos de microtasks. A CC é uma métrica que mede a quantia de caminhos distintos que um software pode executar a partir de um código fonte. Adotar CC em projetos CS pode aumentar consideravelmente o tamanho de um projeto, visto que as microtasks são porções reduzidas de trabalho que devem ser testadas individualmente durante a integração do projeto. As métricas Mean Time Between Failures (MTBF) e suas variações (Mean Time Between System Aborts (MTBSA), Mean Time Between Critical Failures (MTBCF), Mean Time Between Unscheduled Removal (MTBUR) e Mean Time To Failure (MTTF)) só podem ser aplicadas em projetos concluídos, pois aferem com base nas falhas, abortos, erros críticos ou remoção de um sistema em execução. Cenário pouco eficiente para ser aplicado em ambientes CS já que os projetos encontram-se particionados em diversas microtasks.

Portanto, além da escassez de estudos sobre complexidade de execução de microtask, as métricas atuais incorporam particularidades dos ambientes em que foram concebidas e não abrangem as características dos ambientes CS. Amparados nessas lacunas, este trabalho gerou um método para calcular a complexidade de uma microtask. Para realizar a avaliação de tal método foi conduzido um estudo de caso em uma plataforma de desenvolvimento de software CS.

O restante deste trabalho encontra-se organizado da seguinte forma: a próxima seção apresenta os trabalhos relacionados, seguido pela revisão bibliográfica dos temas abordados. O método para determinar a complexidade da microtask é apresentado na seção 4 e logo após a sua avaliação. Os resultados são discutidos na seção 6 e conclusões são apresentadas no final deste artigo.

\section{Trabalhos Relacionados}

Existem diversas contribuições na literatura com objetivos sinérgicos ao desta pesquisa. Desse modo, é necessário explorar e revisitar o que já foi pesquisado e produzido. Logo, o objetivo desta seção é analisar trabalhos semelhantes exibindo suas contribuições e também limitações.

Inicialmente, é pertinente destacar a recente contribuição de [Jacques 2018] que explorou a fronteira que abstrai uma microtask de uma macrotask com base na complexidade de execução. A pesquisa do autor serviu como um importante marco para avançar a literatura sobre complexidade, todavia, a sua contribuição é restrita a sistematização do conhecimento existente, limitando-se em uma abordagem teórica. Ademais, o foco do trabalho também é restrito ao fornecer apenas intuições sobre o design e estrutura das atividades CS, não focando efetivamente na determinação da complexidade.

O trabalho desenvolvimento pelos autores [Aipe e Guadiraju 2018] revelou que a complexidade é um dos principais elementos que caracterizam e afetam a execução de uma atividade CS. Todavia, os autores direcionaram o foco do trabalho na investigação de semelhanças entre as atividades, reduzindo o impacto das contribuições sobre a 
complexidade. Além do mais, o trabalho se apoiou em uma equação matemática para estabelecer o grau de semelhança tornando obtuso aos leitores a compreensão de como foi extraído o grau de complexidade.

Além destes estudos também é importante evidenciar o trabalho de [Yang et al. 2016] que investigou como a complexidade das atividades CS é percebida pelos participantes anônimos. Em resumo, o trabalho dos autores buscou detectar como a complexidade de uma atividade CS afetava a sua execução. Apesar do avanço proporcionado na literatura o trabalho possui uma série de limitações, como a dificuldade de generalização de resultados para o desenvolvimento de software CS (as atividades investigadas eram oriundas de diversas áreas, como tradução de textos e imagens) e as limitações da abordagem empregada (que focou somente na descrição e ignorou outros elementos, como o tempo disponível e o total de tecnologias empregadas nas microtasks).

Por fim, [Nakatsu et al. 2014] desenvolveram uma taxonomia do CS baseado na complexidade das atividades. A taxonomia, apesar de ampla, apresenta algumas limitações de aplicação prática no campo de desenvolvimento de software. Nesse sentido destaca-se a falta de atualização no estudo, a fragilidade em sua estrutura que considerou apenas três dimensões e a dificuldade de generalizar os resultados para o desenvolvimento de software CS.

\section{Revisão Bibliográfica}

Esta seção contextualiza os temas tratados por este trabalho. Desse modo é brevemente sumarizado o funcionamento do CS, a forma como o CS é aplicado no desenvolvimento de software e o como são organizadas e estruturadas as microtasks.

\subsection{Crowdsourcing}

O termo CS surgiu no ano de 2006 para apresentar um novo modelo de negócios que estava emergindo no cenário produtivo. De acordo com os criadores do termo, Jeff Howe e Mark Robinson, diversas empresas estavam modificando o seu eixo tradicional de desenvolvimento terceirizando funções (antes realizadas por seus colaboradores) para uma rede anônima de pessoas [Howe 2006b]. É importante destacar que o CS era uma personalização de um modelo bem estruturado e conhecido, o outsourcing. Todavia, como [Howe 2006a] destacou, a principal diferença entre CS e o outsourcing residia na realização de uma chamada aberta (campanha ou convite flexível) buscando atrair uma potencial rede de participantes anônimos.

Apesar dos riscos na participação anônima o CS demonstrou grande êxito de uso em diversas áreas da sociedade, como por exemplo, na astronomia, área em que o CS possibilitou a recente descoberta de um sistema multiplanetário [Greicius 2018]; ou na geografia, campo que o CS vem influenciando a produção de conhecimento [Sui et al. 2012]; ou ainda na Engenharia de Software, setor que está aplicando o CS para as etapas de design, desenvolvimento e teste de software [Mao et al. 2017].

O modelo de funcionamento do CS é praticamente o mesmo em todos os setores aos quais vem sendo utilizado. Em resumo, uma organização possui um projeto a ser desenvolvido, divide-o em uma lista de atividades a serem realizadas e cadastra essa coleção de atividades em um site ou plataforma online. Em seguida os participantes interessados em desenvolver alguma atividade submetem suas soluções. A organização, 
ao final de um prazo estabelecido, seleciona a(s) melhore(s) solução(ões) e retribui os seus criadores por meio de uma premiação. Observe na Figura 1 uma abstração deste processo.

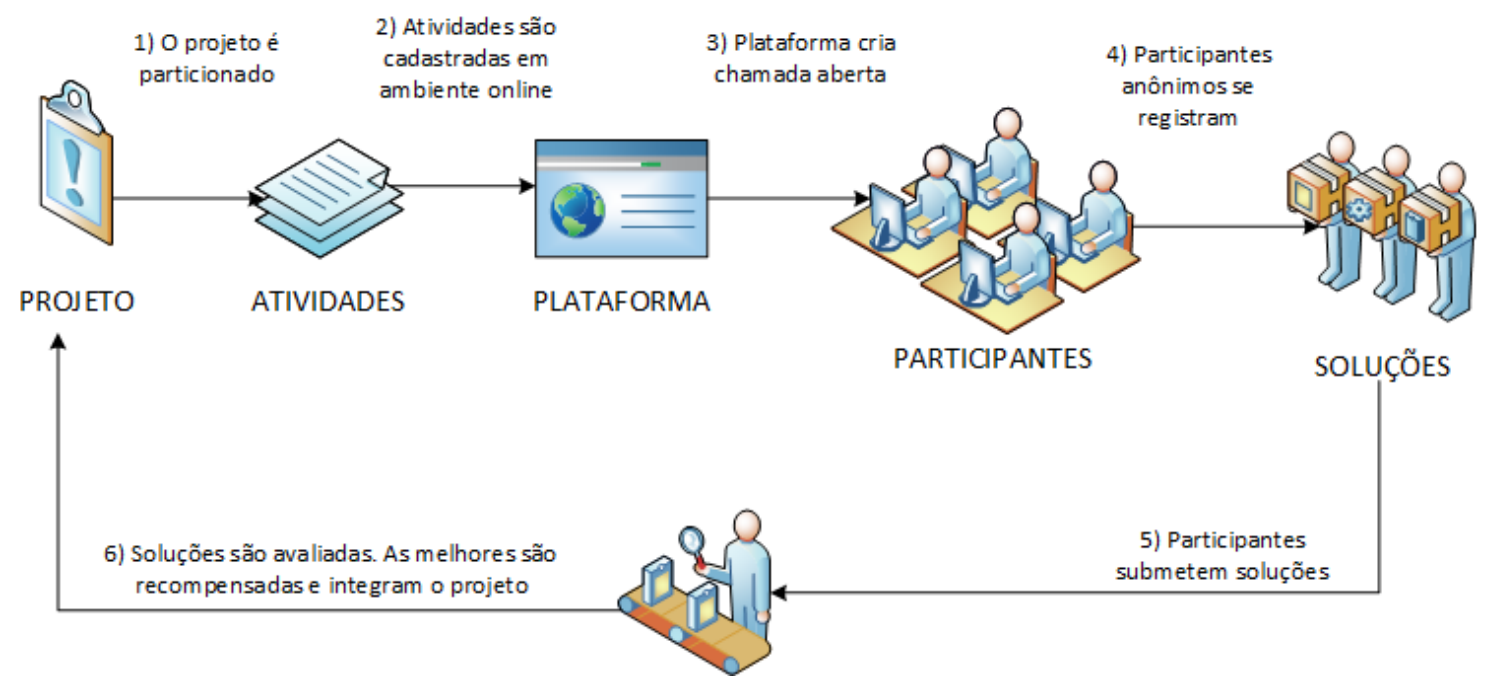

Figura 1. O funcionamento básico do crowdsourcing

Apesar da natureza dinâmica e anônima referente ao funcionamento do CS diversos benefícios são produzidos, como a flexibilidade geográfica e horária, a dispensa de trâmites burocráticos para contratação de profissionais e o acesso a uma extensa amplitude de participantes com habilidades distintas [Abhinav et al. 2018] e [Kurve15 et al. 2018]. Todos esses fatores acabaram impulsionando o CS em diferentes formas de utilização, principalmente no desenvolvimento de software. Assim, é pertinente elucidar sobre tal aplicação.

\subsection{Desenvolvimento de Software Crowdsourcing}

De acordo com [Mao et al. 2017], o desenvolvimento de software CS é o ato de executar qualquer atividade do ciclo de vida de um projeto por meio um grupo anônimo e potencialmente grande de participantes formado por meio de uma chamada aberta.

Para isso, o desenvolvimento de software CS geralmente envolve três tipos de atores: o crowdsourcer, que é o líder técnico e responsável pelo projeto; os crowd workers, que são os participantes anônimos; e a plataforma, sistema online acessado pelo crowdsourcer e pelos crowd workers.

Apesar das semelhanças com outros modelos de desenvolvimento, o CS apresenta um conjunto amplo de características que tornam seus desafios únicos quando comparados aos outros modelos de desenvolvimento de software [Li et al. 2015].

Nesse sentido, destaca-se que o gerenciamento do CS é praticamente inexistente visto que não existem mecanismos para acompanhamento do trabalho dos crowd workers. Além disso, o CS emprega participação colaborativa (todos procurando erros ou otimizações) ou competitiva (primeira submissão aprovada é considerada vencedora). $\mathrm{O}$ processo de desenvolvimento do CS é fracamente aberto, visto que ele é necessário possuir o cadastro em alguma plataforma CS para ter acesso as microtask disponíveis. Por fim, as atividades representam vetores opostos de funcionamento. No open source a comunidade define o que deve ser feito, enquanto que no outsourcing líder e equipe 
selecionam as atividades, e no CS apenas o líder do projeto seleciona o trabalho a ser desenvolvido, sem consultar os participantes. A Tabela 1 resume as principais diferenças entre os modelos similares de desenvolvimento e o modelo CS.

Tabela 1. Comparação Entre Modelos de Desenvolvimento de Software Baseado em [Li et al. 2015] e [Naik et al. 2016]

\begin{tabular}{lccc}
\hline & Open source & Outsourcing & Crowdsourcing \\
\hline Gerenciamento & $\begin{array}{c}\text { Colaboração com } \\
\text { ferramentas online }\end{array}$ & Ferramentas online & $\begin{array}{c}\text { Praticamente } \\
\text { inexistente }\end{array}$ \\
\hline Participação & Colaborativa & Times definidos & $\begin{array}{c}\text { Colaborativa ou } \\
\text { competitiva }\end{array}$ \\
\hline Processo & Aberto & Fechado & Fracamente aberto \\
\hline Atividades & Comunidade define & $\begin{array}{c}\text { Líder e equipe definem } \\
\text { por contrato }\end{array}$ & Líder define o contrato \\
\hline
\end{tabular}

\subsection{Microtasks}

As microtasks são atividades de porções reduzidas executadas no desenvolvimento de software CS, todavia, a literatura concentra um amplo dicionário de termos distintos, como "tasks", "tasks crowd", "minitasks" entre outras variações [Karim et al. 2018, Winkler et al. 2017, Hosseini et al. 2014] e [Mao et al. 2013]. Apesar das opções usadas, o termo microtask é o mais conhecido e difundido em grande parte dos estudos da área. Assim, para facilitar a compreensão do tema e evitar dúvidas deste sentido este trabalho adotou o termo "microtasks" para designar as atividades que são executadas no desenvolvimento de software CS.

Um exemplo de microtask aplicada ao desenvolvimento de software CS é encontrado na Figura 2, captada em uma plataforma CS para ilustrar a sua estrutura básica. Essencialmente existem três grupos de informações na microtask: descrição (contendo instruções de execução), perfil de crowd workers (neste caso, restrito aos trabalhadores que possuem determinados navegadores e sistemas operacionais) e o valor de retribuição .

Segundo [LaToza e Hoek 2015] as microtask representam a menor porção de trabalho possível. Entretanto, é praticamente impossível afirmar que todas são simples e representam apenas uma única porção de trabalho. Quando se põem em perspectiva o desenvolvimento de software, com atividades que possuem interdependência, é uma utopia dizer que todas as tarefas estão completamente atomizadas e reduzidas.

Apesar disso, as microtasks possuem grande portabilidade de aplicação. Isso é evidenciado pela crescente diversidade de múltiplas plataformas CS. Por exemplo, a MKTurk suporta microtasks de múltiplas naturezas, variando desde a usabilidade de sistemas até o processamento de dados. Já a Samasource é uma plataforma em que crowdsourcers enviam projetos para serem "quebrados" em microtasks. Além dessas plataformas, existem outros indicadores que demonstram o crescente emprego do conceito de micro atividades. Nesse sentido é importante destacar as considerações de [Goke e Freitag 2014] que mostraram o crescimento do interesse de diversas empresas, como a IBM, Google e BMW, que atualmente estão solucionando desafios por meio de microtasks. 


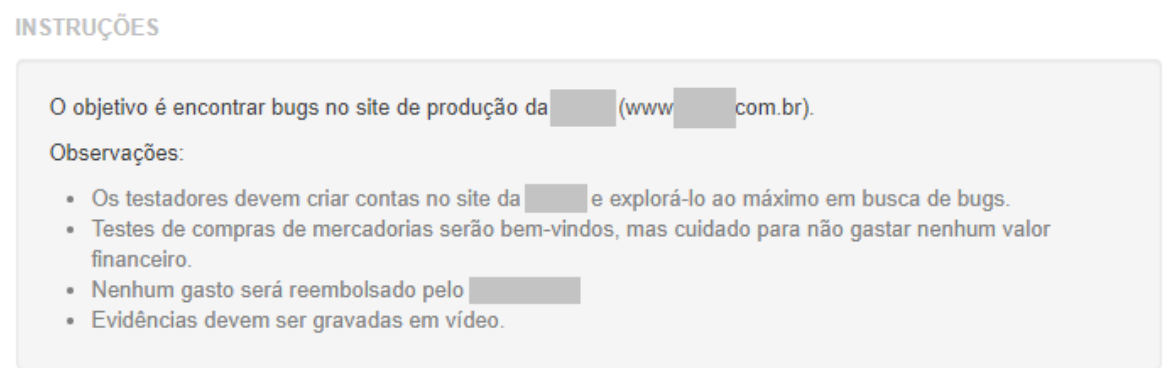

\section{PERFIL DOS TESTADORES}

Navegadores

$$
\begin{aligned}
& \text { - Chrome } 64 \\
& \text { - Chrome } 65 \\
& \text { - Chrome } 66 \\
& \text { - Chrome } 67 \\
& \text { - Chrome } 68 \\
& \text { - Chrome } 69 \\
& \text { - Chrome } 70 \\
& \text { - Firefox } 57 \\
& \text { - Firefox } 58 \\
& \text { - Firefox } 59 \\
& \text { - Firefox } 60 \\
& \text { - Firefox } 61
\end{aligned}
$$

VALORES DAS OCORRÊNCIAS
- OS X 10.10 Yosemite

- OS X 10.11 El Capitan

- OS X 10.12 Sierra

- OS X 10.13 High Sierra

- Windows 10

- Windows 7

- Windows 7 64-bits

- Windows 8

- Windows 8 64-bits

- Windows 8.1

- Windows 8.164-bits

Figura 2. Exemplo de uma Microtask

Finalmente, também é importante destacar que as microtasks exercem múltiplas influências no desenvolvimento de software CS. O crowdsourcer é o responsável técnico e por isso realiza a definição e o cadastro da microtask, além de fiscalizar a sua execução. Já a plataforma é responsável pelo processo de submissão, que engloba a realização da chamada aberta para atrair participantes, suportar o registro e soluções. Por fim, existem os crowd workers que são os trabalhadores que executam e enviam soluções para as microtasks.

\section{Método de Determinação de Complexidade}

O objetivo desta seção é apresentar o método proposto neste trabalho para determinar a complexidade de uma microtask. Para isso a seção encontra-se estruturada em duas partes: na primeira porção ocorre uma conceptualização literária apresentando o processo de investigação sobre como a complexidade das microtasks é estimada; na segunda porção é exposta a estruturação, modelagem e aplicação do método proposto.

\subsection{Definindo Complexidade}

Definir efetivamente o que é a "complexidade" em uma microtask pode ser uma tarefa difícil e sinuosa. Isso ocorre em virtude de três fatores principais: primeiro, o ambiente dinâmico das próprias microtasks, que podem representar tarefas de design, desenvolvimento e teste de software; segundo, pela ausência de regulamentação nas plataformas de desenvolvimento de software CS, pois como foi apresentado na Tabela 1, 
o gerenciamento em tais projetos é praticamente inexistente, faltando mecanismos que abstraiam o que é ou não a complexidade; e terceiro, a participação anônima do CS que torna obtuso a realização dos feedbacks por parte dos crowd workers. Desse modo, para evitar discussões pouco fecundas sobre o que vem a ser a complexidade de uma microtask este trabalho procurou por meios próprios contextualizá-la.

As dificuldades anteriormente citadas motivaram os autores deste estudo a realizarem uma investigação manual em trabalhos que tratavam o tema, buscando minerar informações em torno da complexidade das microtasks. Como resultado desta busca percebeu-se que as publicações analisadas (ver [Jacques 2018, Dubey et al. 2016, LaToza e Hoek 2015] e [Tranquillini et al. 2015]) apontavam para um caminho comum em torno do tema: as características de uma microtask. Em suma, notou-se que todas - ou pelo menos grande parte das microtasks - possuem características similares que podem ser quantificadas tornando a complexidade mais evidente. Observe na Tabela 2 uma síntese das características que foram captadas durante as análises dos estudos citados.

Tabela 1. As características identificadas na literatura sobre microtasks

\begin{tabular}{lll}
\hline ID & Nome & Escala \\
\hline 01 & Requisitos & Um para muitos \\
02 & Tecnologias & Uma para muitas \\
03 & Tempo disponível & Minutos para semanas \\
04 & Limitação & Nenhuma para muitas \\
05 & Expertise & Nenhuma para muitas \\
06 & Dependência & Nenhuma para muitas \\
\hline
\end{tabular}

Deve-se alertar que o campo id foi adicionado apenas para identificar as características, não servindo como base para estabelecer nenhum tipo de hierarquia ou prioridade. A seguir ocorre uma breve descrição de cada item identificado:

- 01 - Requisitos: As microtasks possuem um conjunto de requisitos a serem completados. Somente quando todos os requisitos são executados e concluídos uma microtask é considerada finalizada [Dubey et al. 2016]. Além disso, [Jacques 2018] também lembra que conforme maior a lista de requisitos maior será a sua complexidade.

- 02 - Tecnologias: Refere-se à quantia de tecnologias (linguagens de programação, bibliotecas, etc) que uma microtask necessita para ser implementada [Dubey et al. 2016]. O nível de complexidade de uma microtask aumenta em similaridade ao seu número de tecnologias.

- 03 - Tempo disponível: Ao registrar uma microtask o líder deve selecionar o período disponível para a sua conclusão [Dubey et al. 2016, LaToza e Hoek 2015]. Com isso, o tempo disponível para a finalização deve ser considerado como um fator de complexidade. É importante destacar que no modelo CS o tempo disponível demonstra a seguinte lógica: menos tempo reflete em dificuldades para encontrar participantes qualificados. Ou seja, uma microtask que possui apenas 60 minutos para ser executada é considerada mais complexa que uma com 3 dias de execução. Isso ocorre pelo fato da segunda microtask possuir uma vitrine maior de tempo para ser encontrada e selecionada por um crowd worker. Ademais, 
grande parte das plataformas CS não empregam mecanismos para gerenciar a granularidade de uma microtask. Assim, apenas o líder define o prazo de execução. Na prática isso representa que uma atividade com maior tempo disponível não é, obrigatoriamente, uma tarefa mais complexa de ser executada.

- 04 - Limitação: [Dubey et al. 2016] e [Tranquillini et al. 2015] alertam sobre as microtasks que possuem limitações para participantes, como mínimo de experiência, fluência em um determinado idioma, etc. Similar as tecnologias, conforme maior o número de limitações, mais complexa a microtask será considerada.

- 05 - Expertise: Algumas microtasks podem requerer o domínio de algum conhecimento (softwares de edição, componentes específicos, etc) para serem desenvolvidas [LaToza e Hoek 2015, Tranquillini et al. 2015]. Desse modo, as microtasks que demandam um nível de expertise apresentam complexidade maior.

- 06 - Dependência: Segundo [Jacques 2018] e [LaToza e Hoek 2015], existem microtasks que alteram o fluxo, a entrada, a saída ou o processamento de outras tarefas/rotinas. Desse modo, o nível de dependência contribuiu para aumentar a complexidade de uma microtask.

As características foram classificadas em três diferentes escalas: i) um(a) para muitos(as), ii) minutos para semanas e nenhum para muitos. No caso das características 01 e 02 sempre haverá ao menos um requisito e uma tecnologia associada a cada microtask por serem considerados obrigatórios. Na característica 03 a contagem ocorre por meio da duração em minutos até semanas. Por fim, as características 04, 05 e 06 são opcionais, podendo estar ou não presentes nas microtasks, sendo que a ausência de tais características demanda mais simplicidade para a execução.

\subsection{Conversão}

Após compreender como as características atuam em uma microtask foi necessário realizar um procedimento de quantificação. Para isso foi gerado um método que converte e quantifica as características, determinando a complexidade da microtask.

De acordo com a literatura consultada e discutida na subseção anterior, as duas primeiras características, requisitos e tecnologias, variam entre 1 e N para cada microtask enquanto as características referentes as limitações, expertises e interdependências variam entre 0 e N. Com isso, a inclusão de alguma dessas características adiciona um peso +1 ao cálculo de complexidade da microtask. Para parametrizar a característica restante, o tempo disponível, foram criadas 5 escalas: i) microtasks com duração maior ou igual a 30 dias $(+1)$, ii) microtasks com duração até 30 dias (+2), iii) microtasks com duração até 7 dias (+3), iv) microtasks com duração de até um dia (+4) e v) microtasks com até 60 minutos de duração $(+5)$. Essas escalas foram geradas com base na percepção que o tempo disponível atua como um fator de complexidade na microtask, como discutido anteriormente.

Para facilitar a compreensão da conversão, a Figura 3 ilustra o procedimento completo de conversão. A imagem apresenta que a estrutura de uma microtask descende de 6 características. Por sua vez, cada característica possui algum tipo de variação, sendo 
de 1 para $\mathrm{N}$ (requisitos e tecnologias), minutos para semanas (tempo disponível) e 0 para $\mathrm{N}$ (limitação, expertise e dependência).

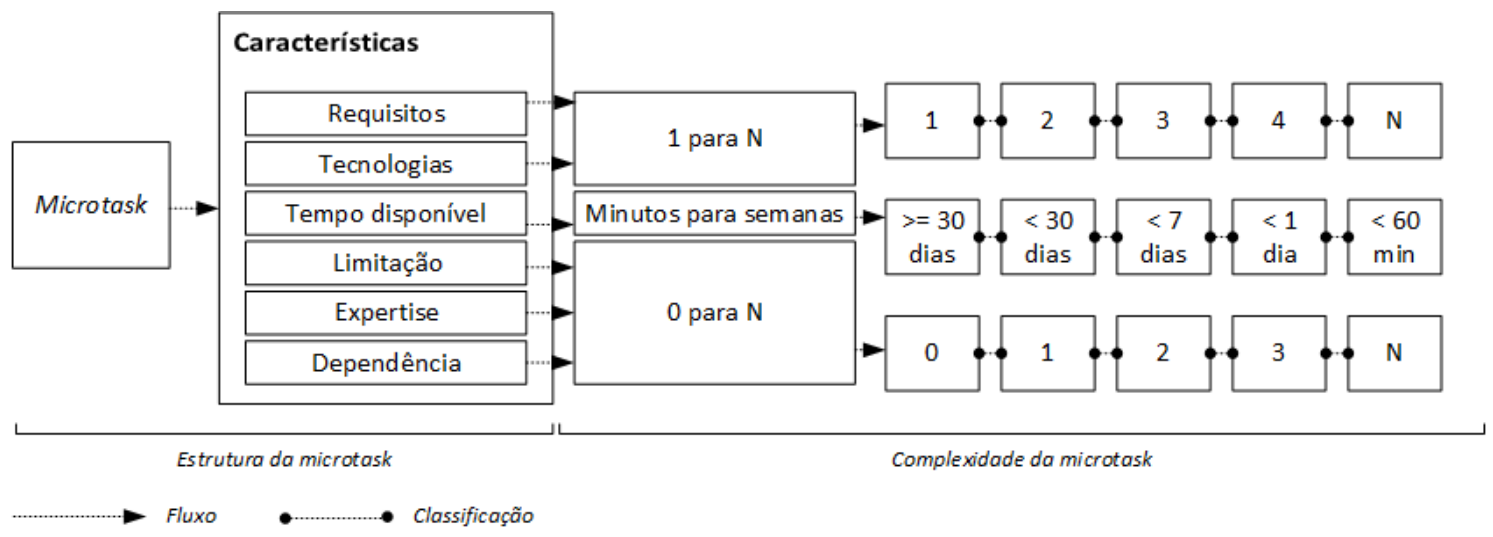

Figura 3. Procedimento para converter características das microtasks em complexidade

O método proposto identifica e classifica seis características de uma microtask. Após isso, cada característica deve ser quantificada por meio de suas ocorrências. Ao fim, a soma total das características determina a complexidade da microtask.

\section{Estudo de caso}

Para avaliar o método de determinação de complexidade proposto neste trabalho foi conduzido um estudo de caso em uma plataforma de desenvolvimento de software CS. Assim, foi abordada a complexidade das microtasks dentro de seu contexto de uso, retratando-as como um elemento essencial e intrinsecamente necessário para que o cenário de CS exista. Para conduzir o estudo de caso foi utilizado os conceitos apresentados por [Yin 2015] e as considerações de [Wohlin et al. 2012]. As próximas subseções descrevem a organização e a configuração do estudo de caso conduzido.

\subsection{Questões de Pesquisa}

A primeira etapa do estudo de caso foi definir as questões de pesquisa (QPs) a serem solucionadas para avaliar a eficácia o método proposto. Desse modo, foram estabelecidas duas seguintes QPs:

- $\left(\boldsymbol{Q P}_{\imath}\right)$ A complexidade de uma microtask influenciou a sua porcentagem de finalização?

- $\left(\boldsymbol{Q P}_{2}\right)$ A complexidade de uma microtask influenciou a quantidade de registros $e$ de submissões?

A teoria mostra que o ambiente CS possui diversas restrições, como a dificuldade de comunicação e ausência de reuniões entre crowd workers, que dificultam a compreensão e a execução de atividades com maior grau de complexidade. Assim, a QP foi elaborada para avaliar se o método proposto seria capaz de identificar como a complexidade de uma microtask poderia influenciar a sua finalização. Em suma, para avaliar sua eficiência, as microtasks consideradas simples pelo método deveriam ter uma porcentagem de finalização superior as microtasks consideradas complexas. 
A $\mathrm{QP}_{2}$ explorou como a complexidade de uma microtask poderia influenciar os registros e submissões por parte dos crowd workers. De forma objetiva foi investigado se o método proposto seria eficiente e capaz de revelar alguma diferença do desempenho de registro e submissão entre as microtasks consideradas simples e complexas.

\subsection{Seleção do Caso}

Após definir as QPs a serem analisadas foi iniciado o processo para a seleção de caso. Os critérios definidos para tal seleção foram estipulados de acordo com as seguintes regras:

i) Selecionar apenas organizações que desenvolvam software em ambientes CS;

ii) Selecionar apenas organizações que possam suportar a execução de microtasks de design, desenvolvimento e teste de software.

Deve-se salientar que o primeiro critério foi estabelecido para filtrar apenas as organizações que são o foco deste estudo, cujo propósito é desenvolver software em ambientes CS. Enquanto que o segundo critério filtrou as organizações que suportavam a execução de microtasks em pelo menos três etapas do ciclo de vida de um projeto de software. Dentro desse cenário apenas uma plataforma foi contemplada, denominada neste trabalho como plataforma $\mathrm{X}$, que por fatores estratégicos não possuirá a sua identificação revelada.

A plataforma $\mathrm{X}$ opera como uma das grandes lideranças dentro do mercado de desenvolvimento de software CS, sendo uma das responsáveis por disseminar tal prática.

O lançamento da plataforma X ocorreu no ano de 2001. No início de sua operação apenas realizava competições de programação. Nessa época os participantes se cadastravam na plataforma, selecionavam desafios de programação e enviam soluções próprias. As soluções mais bem avaliadas eram recompensadas por meio de um processo de premiação financeira. Este modelo de competição foi evoluindo conforme o avanço dos anos, e em 2006 com a disseminação do CS a plataforma X diversificou a sua atuação. Nesse período a plataforma começou a oferecer contratos para clientes que desejassem solucionar problemas e desafios próprios, como por exemplo, a execução de atividades focadas em design web e também ideias de aplicações, aumentando assim a sua base de clientes e também a sua comunidade de participantes. Em vista dessa estratégia, a plataforma conseguiu sobressair e crescer exponencialmente dentro do mercado internacional. Atualmente, a plataforma X possui cerca de 1.200 .000 crowd workers. Tais trabalhadores possuem experiência em áreas como design, desenvolvimento, ciência de dados e programação competitiva. Além disso, a plataforma já distribuiu mais de US\$ 80.000.000 em premiação para a sua comunidade por meio da execução de desafios'

Em relação ao seu processo de desenvolvimento, a plataforma $\mathrm{X}$ emprega um conjunto de características e regras para aplicar CS no desenvolvimento de software. De modo simplificado tal processo pode ser dividido em quatro etapas distintas, organizadas da seguinte maneira:

\footnotetext{
Cabe destacar que a plataforma rótula as atividades como "desafios" para tornar o seu produto mais atrativo aos clientes e participantes. Todavia, o termo "desafio" é considerado apenas uma terminologia opcional ao termo "microtask", adotado neste trabalho.
} 
1) Um crowdsourcer cadastra uma microtask na plataforma. Nesse ato, o crowdsourcer deve preencher os seguintes campos: nome, descrição, tipo (design, desenvolvimento ou teste), duração (em dias) e premiação;

a. É importante destacar que não existe fiscalização na etapa de cadastro, ou seja, os crowdsourcers podem cadastrar atividades com informações obtusas, faltantes ou simplesmente impraticáveis;

b. O tempo de duração é estipulado entre duas datas (data inicial e data final), representado o total de dias disponíveis para a execução da microtask. Alcançado a data final a microtask vai para avaliação e não é possível realizar novas submissões;

c. A plataforma possui outras categorias de classificação, não ficando restrita apenas ao design, desenvolvimento e teste de software CS. Contudo, o estudo de caso focou apenas nos tipos investigados.

2) Após o cadastro a microtask fica disponível na plataforma e crowd workers podem se registrar para a execução;

3) Após um crowd worker se registrar, ele possui habilitação para submeter várias submissões até a data final da microtask;

a. Nenhum crowd worker é punido se não submeter soluções.

4) Após a finalização do prazo, o crowdsourcer avalia todas as submissões e seleciona a(s) melhor(es) solução(ões), retribuindo os respectivos crowd workers.

a. Nesta etapa ocorrem dois cenários possíveis: a microtask é finalizada com sucesso (alguma submissão foi escolhida vencedora) ou falha (nenhuma submissão foi escolhida vencedora ou não houve nenhuma submissão).

\subsection{Procedimento de Coleta de Dados}

Para realizar a coleta de dados os autores consultaram a baseline da plataforma X. Tal baseline apresentava uma coleção de microtasks já desenvolvidas, contendo seus metadados (como nome, descrição, categoria, duração, premiação, etc) e status de execução (sucesso ou falha).

Os dados recuperados na consulta da baseline foram organizados e tabulados em uma planilha eletrônica. Cada linha da planilha correspondia a uma microtask e era identificada por um código único.

Após isso, todos os códigos presentes na planilha foram inseridos na ferramenta Random Picker. Essa ferramenta realizava uma escolha aleatória revelando o código de uma microtask. Assim, após cada escolha a respectiva microtask selecionada era analisada para compor este estudo de caso. Naturalmente, o código colhido era removido da lista para novas seleções, evitando que uma microtask fosse selecionada duas ou mais vezes.

Foram realizadas 30 seleções aleatórias durante esse processo de escolha. $\mathrm{O}$ critério de parada para seleção foi definido com base nas seguintes condições: primeiro, a amostra coletada deveria possuir o mesmo número de microtasks com sucesso e falha; segundo, a amostra coletada deveria envolver microtasks de design, desenvolvimento e 
teste na mesma proporção. Ao alcançar 30 seleções as duas condições foram satisfeitas e foi iniciado a próxima etapa do estudo.

\subsection{Procedimento de Análise}

As microtasks selecionadas foram tabuladas em uma nova planilha eletrônica, e com base nessa planilha, foi iniciado o seguinte processo de análise:

- Cada linha da nova planilha correspondia a uma microtask. E cada item possuía uma série de informações coletadas em diferentes domínios, como em campos textuais (nome e descrição da microtask); campos de datas (início e final dos registros); e campos numéricos (total de tecnologias empregadas). Assim, foi necessário analisar os dados individualmente para padronizar as informações em toda a amostra. Durante essa etapa, os autores buscaram extrair e organizar com exatidão as informações referentes aos requisitos, tecnologias, tempo disponível, limitações, expertise e dependência de cada microtask.

- As informações extraídas foram quantificadas conforme as premissas apresentadas na subseção 4.2 deste estudo. Para exemplificação, considere que determinada microtask tivesse apresentada em sua descrição que seria obrigatório completar dois requisitos para sua finalização (exemplo: submeter a solução em extensão .zip e descrevê-la em um arquivo com extensão .txt), a microtask receberia o valor +2 para a característica de requisitos. Esse procedimento foi realizado para todas as características (requisitos, tecnologias, tempo disponível, limitações, expertise e dependência) existentes em cada microtask.

\subsection{Procedimento de Avaliação}

Nesta etapa foram empregados diferentes recursos da estatística descritiva como forma de auxiliar o processo investigativo. No que concerne tal procedimento cabe destacar que foram aplicados cálculos de frequência para compreender a natureza da distribuição dos dados. Além destes, também recorreu-se a aplicação de medidas de dispersão na companhia de técnicas de visualização gráfica para descrever visualmente a disposição de dados e facilitar a sua compreensão. Por fim, cabe destacar que todo o procedimento de avaliação foi realizado com base no uso de um software estatístico denominado

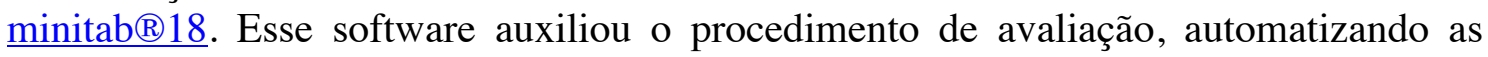
etapas da análise de frequência e de dispersão e gerando automaticamente os gráficos.

Além disso, para reduzir a incidência de riscos, destaca-se que a captação de microtasks foi realizada de modo aleatório, evitando coletar dados viciados ou repetidos. Além disso, todas as microtasks selecionadas foram investigadas manualmente pelos pesquisadores.

\section{Resultados}

O objetivo desta seção é sumarizar os principais resultados obtidos com a execução do estudo de caso. Assim, a seção encontra-se estruturada em cinco porções: a primeira porção mostra uma visão geral da amostra analisada no estudo de caso. A segunda e a terceira partes focam, respectivamente, na análise da complexidade versus o índice de finalização $\left(\mathrm{QP}_{1}\right)$ e na investigação da complexidade versus os registros e as submissões 
$\left(\mathrm{QP}_{2}\right)$ a quarta porção apresenta uma síntese dos resultados obtidos e a última seção aborda as limitações do estudo.

\subsection{Análises iniciais}

O estudo de caso coletou dados de 30 microtasks. Inicialmente, a amostra foi organizada e dividida de acordo com o tipo de sua aplicação (design, desenvolvimento ou teste) e seu status de finalização (sucesso ou falha). A relação que apresenta as frequências e as descrições de cada tipo de microtasks presente na amostra é encontrada na Tabela 3 . Além disso, a frequência referente ao status de finalização está exposta na Tabela 4.

Tabela 3. Visão geral da amostra em relação ao tipo

\begin{tabular}{llcc}
\hline Etapa & Descrição & Total & Frequência \\
\hline Design & protótipos e interfaces & 10 & $33.33 \%$ \\
Desenvolvimento & implementação de códigos & 10 & $33.33 \%$ \\
Teste & correção e busca de erros & 10 & $33.33 \%$ \\
\hline
\end{tabular}

Tabela 4. Visão geral da amostra em relação ao status de finalização

\begin{tabular}{lcc}
\hline Etapa & Sucesso & Falha \\
\hline Design & $50 \%$ & $50 \%$ \\
Desenvolvimento & $50 \%$ & $50 \%$ \\
Teste & $50 \%$ & $50 \%$ \\
\hline
\end{tabular}

Em relação ao status de finalização das microtasks, cabe destacar o conjunto de regras adotadas.

- SUCESSO: para definir uma microtask com sucesso de execução é fiscalizado se todas as seguintes condições foram satisfeitas:

○ Houve ao menos um registro e uma submissão;

○ Houve ao menos uma submissão selecionada;

- A solução selecionada foi enviada até o prazo estipulado;

- A solução selecionada cumpriu todos os requisitos expressos na descrição da microtask.

- FALHA: já para uma microtask possuir o status de cancelada (falha) podem ocorrer as seguintes condições:

○ Não houve nenhum registro ou nenhuma submissão;

○ Nenhuma submissão foi selecionada;

○ As submissões não respeitaram o prazo estipulado ou não possuíam atendido algum requisito;

- A microtask se mostrou inviável por possuir muitos requisitos a serem cumpridos.

Em via dessas considerações a amostra foi dividida igualitariamente (metade com sucesso e a outra metade com falha) para balancear a amostra e evitar a concentração de 
dados em torno de somente um status de finalização. Tal disposição é apresentada por meio da Tabela 5, que evidencia ainda a etapa de execução (design, desenvolvimento ou teste), os registros e as submissões recebidas.

Tabela 5. Visão geral da amostra em relação aos registros e submissões

\begin{tabular}{|c|c|c|c|c|}
\hline Identificador & Etapa & Status & Registros & Submissões \\
\hline 2 & Design & Sucesso & 8 & 2 \\
\hline 4 & Desenvolvimento & Sucesso & 18 & 3 \\
\hline 497 & Teste & Sucesso & 13 & 3 \\
\hline 511 & Design & Sucesso & 14 & 1 \\
\hline 694 & Desenvolvimento & Sucesso & 26 & 4 \\
\hline 804 & Teste & Sucesso & 8 & 1 \\
\hline 806 & Teste & Sucesso & 9 & 4 \\
\hline 1455 & Teste & Sucesso & 12 & 3 \\
\hline 1579 & Teste & Falha & 13 & 0 \\
\hline 2484 & Teste & Sucesso & 7 & 1 \\
\hline 2615 & Desenvolvimento & Falha & 28 & 3 \\
\hline 2897 & Design & Falha & 19 & 2 \\
\hline 3122 & Desenvolvimento & Sucesso & 10 & 2 \\
\hline 3657 & Desenvolvimento & Falha & 10 & 0 \\
\hline 4734 & Design & Sucesso & 10 & 4 \\
\hline 6094 & Design & Falha & 7 & 0 \\
\hline 6356 & Desenvolvimento & Sucesso & 12 & 3 \\
\hline 6968 & Design & Falha & 3 & 0 \\
\hline 8604 & Desenvolvimento & Falha & 23 & 0 \\
\hline 8704 & Desenvolvimento & Falha & 7 & 0 \\
\hline 9354 & Design & Falha & 12 & 0 \\
\hline 9942 & Design & Sucesso & 8 & 3 \\
\hline 10591 & Teste & Falha & 6 & 0 \\
\hline 12257 & Teste & Falha & 17 & 0 \\
\hline 12743 & Design & Sucesso & 7 & 2 \\
\hline 12888 & Desenvolvimento & Sucesso & 43 & 17 \\
\hline 15181 & Teste & Falha & 7 & 0 \\
\hline 15407 & Desenvolvimento & Falha & 13 & 1 \\
\hline 15749 & Teste & Falha & 12 & 0 \\
\hline 15900 & Design & Falha & 7 & 0 \\
\hline
\end{tabular}

A teoria central do método apresentado neste trabalho propõe quantificar as características de uma microtask, obtendo assim a sua complexidade final por meio de uma soma. Nesse sentido, a Tabela 6 mostra a quantificação de cada característica obtida na amostra analisada. Um interessante recorte dessa etapa foi a identificação que a "Expertise" representou a característica que menos vezes foi acionada nas microtasks. Ou seja, em grande parte da amostra, os crowdsourcers não estipularam domínios de nenhum conhecimento aos crowd workers. Enquanto isso, em termos gerais, os "Requisitos" representaram a característica mais utilizada nas microtasks. Essas percepções podem demonstrar certa tendência do próprio CS, já devido ao trabalho anônimo é extremamente difícil para o crowdsourcer comprovar que os crowd workers possuíam realmente as expertises solicitadas. Além do mais, devido a dificuldade de comunicação é comum que os requisitos detalhem com maior objetividade o que de fato deve ser feito para executar a microtask.

Tabela 6. Extração e conversão dos dados das microtasks 


\begin{tabular}{cccccccc}
\hline Identificador & Requisitos & Tecnologias & Tempo & Limitações & Expertise & Dependência & Complexidade \\
\hline 2 & 2 & 1 & 3 & 2 & 0 & 1 & 9 \\
4 & 3 & 4 & 3 & 2 & 0 & 1 & 13 \\
497 & 15 & 1 & 2 & 0 & 0 & 0 & 18 \\
511 & 1 & 2 & 3 & 0 & 1 & 5 & 12 \\
694 & 4 & 1 & 3 & 0 & 0 & 2 & 10 \\
804 & 4 & 1 & 3 & 0 & 0 & 11 & 19 \\
806 & 6 & 1 & 3 & 0 & 0 & 4 & 14 \\
1455 & 4 & 4 & 2 & 0 & 0 & 3 & 13 \\
1579 & 17 & 2 & 3 & 1 & 0 & 1 & 24 \\
2484 & 4 & 4 & 2 & 1 & 0 & 1 & 12 \\
2615 & 12 & 1 & 3 & 1 & 0 & 1 & 18 \\
2897 & 3 & 4 & 3 & 0 & 0 & 5 & 15 \\
3122 & 5 & 6 & 3 & 0 & 0 & 1 & 15 \\
3657 & 4 & 4 & 3 & 6 & 0 & 1 & 18 \\
4734 & 8 & 0 & 3 & 0 & 0 & 4 & 15 \\
6094 & 9 & 2 & 3 & 2 & 0 & 1 & 17 \\
6356 & 7 & 2 & 3 & 1 & 0 & 1 & 14 \\
6968 & 13 & 0 & 3 & 1 & 0 & 0 & 17 \\
8604 & 5 & 1 & 3 & 1 & 4 & 4 & 18 \\
8704 & 6 & 3 & 3 & 1 & 1 & 3 & 17 \\
9354 & 4 & 3 & 3 & 2 & 4 & 1 & 17 \\
9942 & 6 & 1 & 3 & 0 & 0 & 2 & 12 \\
10591 & 27 & 2 & 2 & 7 & 0 & 4 & 42 \\
12257 & 9 & 4 & 2 & 0 & 0 & 13 & 28 \\
12743 & 2 & 1 & 3 & 1 & 1 & 2 & 10 \\
12888 & 7 & 1 & 3 & 0 & 0 & 3 & 14 \\
15181 & 10 & 2 & 2 & 3 & 0 & 5 & 22 \\
15407 & 4 & 2 & 2 & 0 & 0 & 1 & 9 \\
15749 & 2 & 2 & 2 & 2 & 0 & 12 & 20 \\
15900 & 26 & 2 & 3 & 2 & 0 & 0 & 33 \\
\hline
\end{tabular}

Nota: Complexidade $=R+T+T e+L+E+D$, em que $R=$ requisitos, $T=$ tecnologias, $T e=$ tempo, $L=$ limitações, $E=$ expertise e $D=$ dependência.

\subsection{A complexidade da uma microtask influenciou a sua porcentagem de finalização?}

Após realizar a análise inicial dos dados, foi investigada a solução da $\mathrm{QP}_{1}$. Em resumo, o objetivo desta etapa foi verificar se as microtasks mais complexas da amostra foram finalizadas com sucesso ou falha.

Para identificar as microtasks mais complexas foi adotado o cálculo de quartis. De modo resumido pode-se afirmar que os quartis são utilizados para exibir a dispersão e a tendência central de uma amostra. Existem 3 intervalos de quartis, o primeiro quartil (Q1) que representa $25 \%$ dos menores dados encontrados na amostra; o segundo quartil (Q2) que apresenta a mediana amostral e o terceiro quartil (Q3) que exibe os $75 \%$ dos maiores dados amostrais. A relação dos quartis obtidas na analisada amostra é encontrada na Tabela 7.

Tabela 7. Complexidade das microtasks separada entre quartis

\begin{tabular}{lll}
\hline Quartil Condição & Valor \\
\hline
\end{tabular}




\begin{tabular}{lll}
\hline Q1 & Menor ou igual que & 12.75 \\
Q2 & Menor ou igual que & 16.00 \\
Q3 & Menor ou igual que & 18.25 \\
\hline
\end{tabular}

Após a observação dos intervalos entre quartis foi adotado que o Q2 como representaria a fronteira para definir e classificar as microtasks como simples ou complexas. Essa seleção ocorreu em virtude de existirem apenas dois status de finalização (sucesso ou falha). Assim, o Q2 demonstrou ser o valor mais sensato, dividindo a amostra igualitariamente. Ademais, cabe destacar que foi considerado que as microtasks com complexidade menor ou igual a 16 eram simples e que com complexidade superior a 16 foram consideradas complexas, como mostra a Tabela 8.

Tabela 8. Fronteira entre microtasks simples e complexas baseada no segundo quartil amostral

\begin{tabular}{|c|c|c|c|c|}
\hline Classificação & Identificador & Etapa & Status & Complexidade \\
\hline SIMPLES & 15407 & Desenvolvimento & Falha & 9 \\
\hline SIMPLES & 2 & Design & Sucesso & 9 \\
\hline SIMPLES & 12743 & Design & Sucesso & 10 \\
\hline SIMPLES & 694 & Desenvolvimento & Sucesso & 10 \\
\hline SIMPLES & 9942 & Design & Sucesso & 12 \\
\hline SIMPLES & 511 & Design & Sucesso & 12 \\
\hline SIMPLES & 2484 & Teste & Sucesso & 12 \\
\hline SIMPLES & 1455 & Teste & Sucesso & 13 \\
\hline SIMPLES & 4 & Desenvolvimento & Sucesso & 13 \\
\hline SIMPLES & 806 & Teste & Sucesso & 14 \\
\hline SIMPLES & 6356 & Desenvolvimento & Sucesso & 14 \\
\hline SIMPLES & 12888 & Desenvolvimento & Sucesso & 14 \\
\hline SIMPLES & 2897 & Design & Falha & 15 \\
\hline SIMPLES & 4734 & Design & Sucesso & 15 \\
\hline SIMPLES & 3122 & Desenvolvimento & Sucesso & 15 \\
\hline \multicolumn{5}{|c|}{ Fronteira estabelecida por $Q 2$} \\
\hline COMPLEXA & 6094 & Design & Falha & 17 \\
\hline COMPLEXA & 6968 & Design & Falha & 17 \\
\hline COMPLEXA & 8704 & Desenvolvimento & Falha & 17 \\
\hline COMPLEXA & 497 & Teste & Sucesso & 18 \\
\hline COMPLEXA & 8604 & Desenvolvimento & Falha & 18 \\
\hline COMPLEXA & 3657 & Desenvolvimento & Falha & 18 \\
\hline COMPLEXA & 2615 & Desenvolvimento & Falha & 18 \\
\hline COMPLEXA & 804 & Teste & Sucesso & 19 \\
\hline COMPLEXA & 15749 & Teste & Falha & 20 \\
\hline COMPLEXA & 15181 & Teste & Falha & 22 \\
\hline COMPLEXA & 1579 & Teste & Falha & 24 \\
\hline COMPLEXA & 12257 & Teste & Falha & 28 \\
\hline COMPLEXA & 15900 & Design & Falha & 33 \\
\hline COMPLEXA & 10591 & Teste & Falha & 42 \\
\hline
\end{tabular}

As microtasks consideradas simples obtiveram um desempenho de finalização superior em relação as complexas. Essa diferença foi próximo à casa de $90 \%$ de finalização com sucesso. Da amostra analisada, 13 microtasks $(86,67 \%)$ consideradas simples foram finalizadas com sucesso e apenas $2(13,33 \%)$ não obtiveram êxito na finalização. Essa porção é inversamente proporcional ao índice de finalização das 
microtasks complexas, que tiveram apenas $2(13,33 \%)$ finalizadas com sucesso, ao ponto que $13(86,67 \%)$ não foram concluídas, como sintetizado na Tabela 9.

Tabela 9. Complexidade das microtasks e índice de finalização

\begin{tabular}{lccc}
\hline Complexidade & Sucesso & Falha & Finalização (\%) \\
\hline SIMPLES $(<=16)$ & 13 & 2 & $86,67 \%$ \\
COMPLEXAS $(>16)$ & 2 & 13 & $13,33 \%$ \\
\hline TOTAL & $\mathbf{1 5}$ & $\mathbf{1 5}$ & $\mathbf{1 0 0 , 0 0 \%}$ \\
\hline
\end{tabular}

Apesar dos indicadores positivos que demonstraram a aderência do método proposto para detectar sucesso de finalização das microtasks simples das microtasks complexas, 4 amostras despertaram a atenção. Sobre isso, os autores investigaram mais profundamente as microtasks 15407 e 2897 que não foram concluídas com êxito, mas ainda assim foram consideradas simples; e as microtasks 497 e 804, que apesar de serem classificadas como complexas foram finalizadas com sucesso.

15407: Essa microtask possuía o objetivo de providenciar a implementação de um framework para validar e converter documentos. A descrição da microtask estava dentro de um padrão aceitável de detalhes, com um prazo de tempo plausível e com instruções claras e precisas. Todavia, ao aprofundar a investigação ficou evidente qual o motivo da falha de execução: cancelada na revisão. Em suma, isso quer dizer que a microtask era simples e obteve submissões, todavia, nenhuma submissão seguiu corretamente os requisitos especificados e por isso foi considerada falha.

2897: A finalidade dessa microtask era oferecer uma solução para realizar uma integração entre dois sistemas. Similar a microtask anteriormente analisada, essa também contava com uma descrição clara e com tempo compatível. E, similarmente, ocorreu o mesmo motivo de falha, a de ser reprovada na revisão.

Após observar os dois cenários das microtasks ficou evidente que o método proposto foi assertivo por considera-las simples. Assim, as falhas de finalização não se restringiram necessariamente na complexidade, mas sim na falta de atenção dos crowd workers em relação aos requisitos de cada tarefa.

497: Essa microtask foi classificada como complexa e ainda assim obteve sucesso na sua finalização. A sua complexidade se deu, principalmente, no total de requisitos necessários (15), fato que não impediu a sua conclusão exitosa. Dos fatores observados o que se mostrou mais eficiente em explicar tal comportamento foi o foco ao qual a microtask estava condicionada. Tal tarefa era de busca por erros, e isso pode explicar a facilitação de conclusão já que os crowd workers deveriam apenas identificar erros. Apesar de possuir uma alta quantia de requisitos, a busca de erros é mais simples que as atividades de outras etapas, como a criação de protótipos (design), a codificação (desenvolvimento) e a correção de erros (teste), pois tende a ser executada rapidamente.

804: Apesar dos esforços em identificar qual o fator que culminou em classificar essa microtask como complexa e ainda assim ser finalizada com sucesso, não foi obtido nenhuma hipótese eficiente. A microtask 804 possuía muitas dependências (11), um prazo considerado curto ( 3 dias) e ainda assim foi finalizada com sucesso.

Ao fim percebeu-se que o método proposto foi pouco eficiente em classificar essas duas microtasks. Apesar disso, tal consideração ainda não invalidou o método proposto 
(que alcançou quase $90 \%$ de sucesso), mostrando possíveis melhorias a serem desenvolvidas.

\subsection{A complexidade de uma microtask influenciou a quantidade de registros e submissões?}

Como foi dito anteriormente na seção 5.2 deste estudo, a plataforma X emprega duas etapas na execução de microtasks: na primeira etapa os participantes interessados se registram, e na segunda etapa os participantes submetem as suas soluções. Assim, naturalmente o número de submissões tende a ser menor que o número de registros. Tendo isso em vista foi elaborada a $\mathrm{QP}_{2}$ e o objetivo desta subseção é analisar se existe alguma relação entre o índice de registro/submissão e a complexidade que estipulada pelo método proposto. O que se deseja com tal conjectura é identificar se as microtasks mais complexas tiveram menos submissões que as microtasks mais simples.

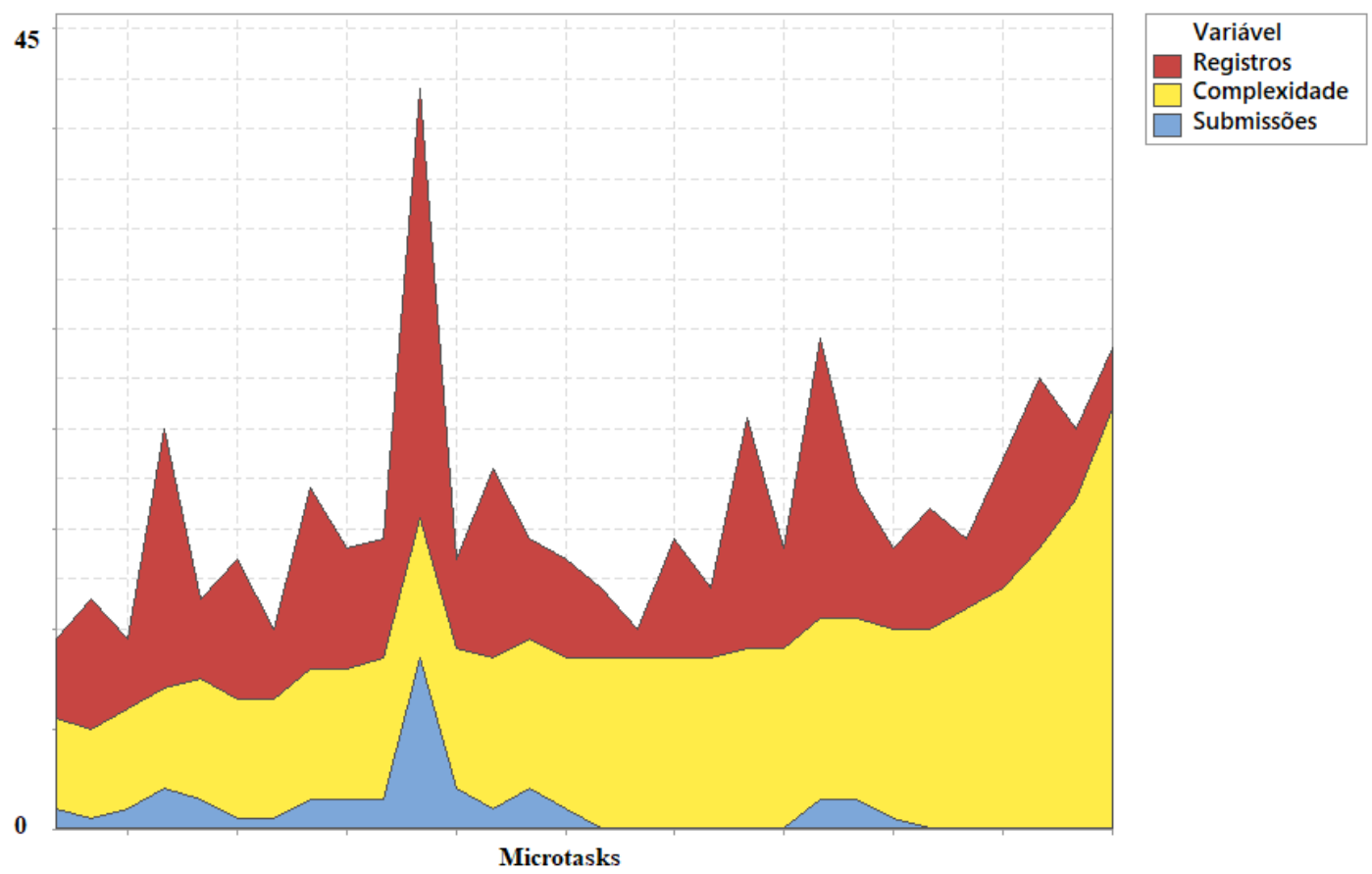

Figura 4. Relação entre complexidade e registros/submissões das microtasks

O ponto de partida da investigação foi visualizar graficamente o comportamento entre a complexidade, os registros e as submissões das microtasks. Para isso foi gerado um gráfico de dispersão sobre os índices analisados, como mostrou a Figura 4.

Ao analisar o gráfico ficou evidente que a complexidade seguiu um padrão bem similar aos registros e submissões. Em suma, o método proposto foi capaz de adequar a dificuldade com o índice de crowd workers que se registraram e submeteram soluções. Em relação a isso, pode-se fazer as seguintes observações:

- A área vermelha destacada no gráfico representa o índice de registros. E na análise inicial deste índice não mostrou nenhum comportamento lógico. Um dos principais fatores que justificam essa observação é o fato das microtasks apresentam picos e reduções de registros em diversos pontos. 
- Já a área em azul apresentou a relação de submissão. Ao analisar essa visão, percebe-se que as submissões estão concentradas na zona a esquerda do gráfico, ao ponto que existe apenas uma pequena ilha na zona a direita que mostra microtasks com submissões.

- Por fim, a área amarela exibiu a complexidade das microtasks, e ao unificar a visão das três áreas notou-se dois comportamentos importantes: o primeiro representa o fato da complexidade seguir o índice de registros, destacando a aderência do método. O segundo apresenta que conforme houve um acréscimo da complexidade houve redução de submissão. Ou seja, naturalmente ao aumentar a complexidade da microtask ocorreu menos submissão.

Tendo em vista as interpretações anteriores, a análise foi aperfeiçoada gerando um gráfico de dispersão (encontrado na Figura 5). Esse gráfico foi separado em quatro quadrantes distintos, que representam uma matriz de complexidade versus registros.

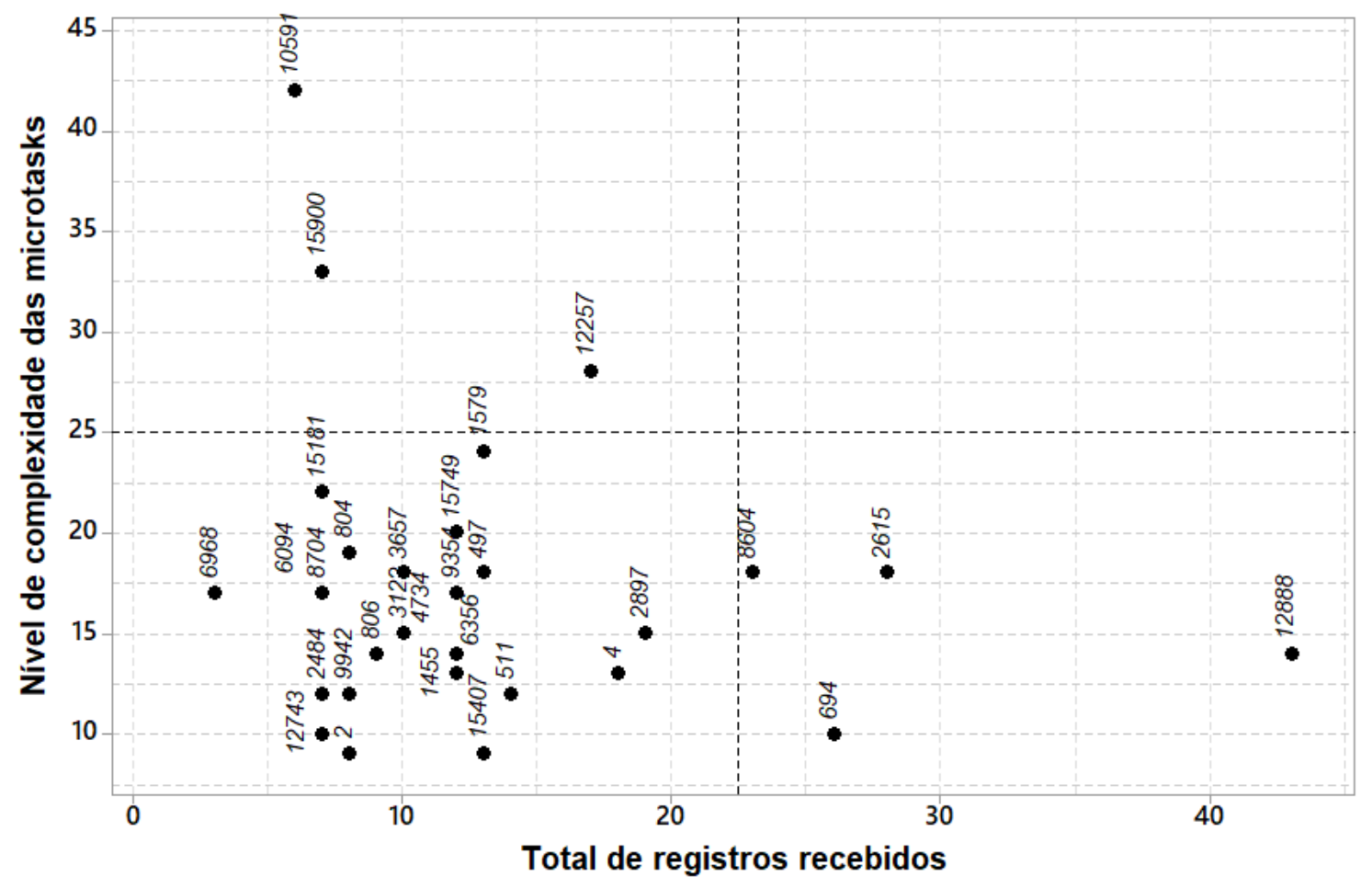

Figura 5. Dispersão entre complexidade e registros das microtasks

Percebeu-se que os dois quadrantes inferiores - esquerdo e direito - concentraram o maior índice de registros de microtasks. Não obstante, esses quadrantes representaram a disposição das microtasks consideradas mais simples mostrando que tarefas com menor grau de dificuldade receberam organicamente mais registros que as atividades complexas.

Os quadrantes superiores - esquerdo e direito - mostram a incidência de apenas três registros. Isso exibiu que as microtasks mais complexas, além de possuírem menor atenção dos crowd workers, não conseguiram ultrapassar cerca de 20 registros. Isso é exposto pelo quadrante superior direito, que ficou vazio, expondo que não houve nenhuma ocorrência de microtask complexa com mais de 20 registros.

Após analisar o índice de registro foi analisado o índice de submissão. O que se buscou observar foi identificar se as microtasks classificadas como simples receberiam 
mais submissões que as microtasks consideradas complexas, e o gráfico de dispersão que ilustra esse diagnóstico é encontrado na Figura 6.

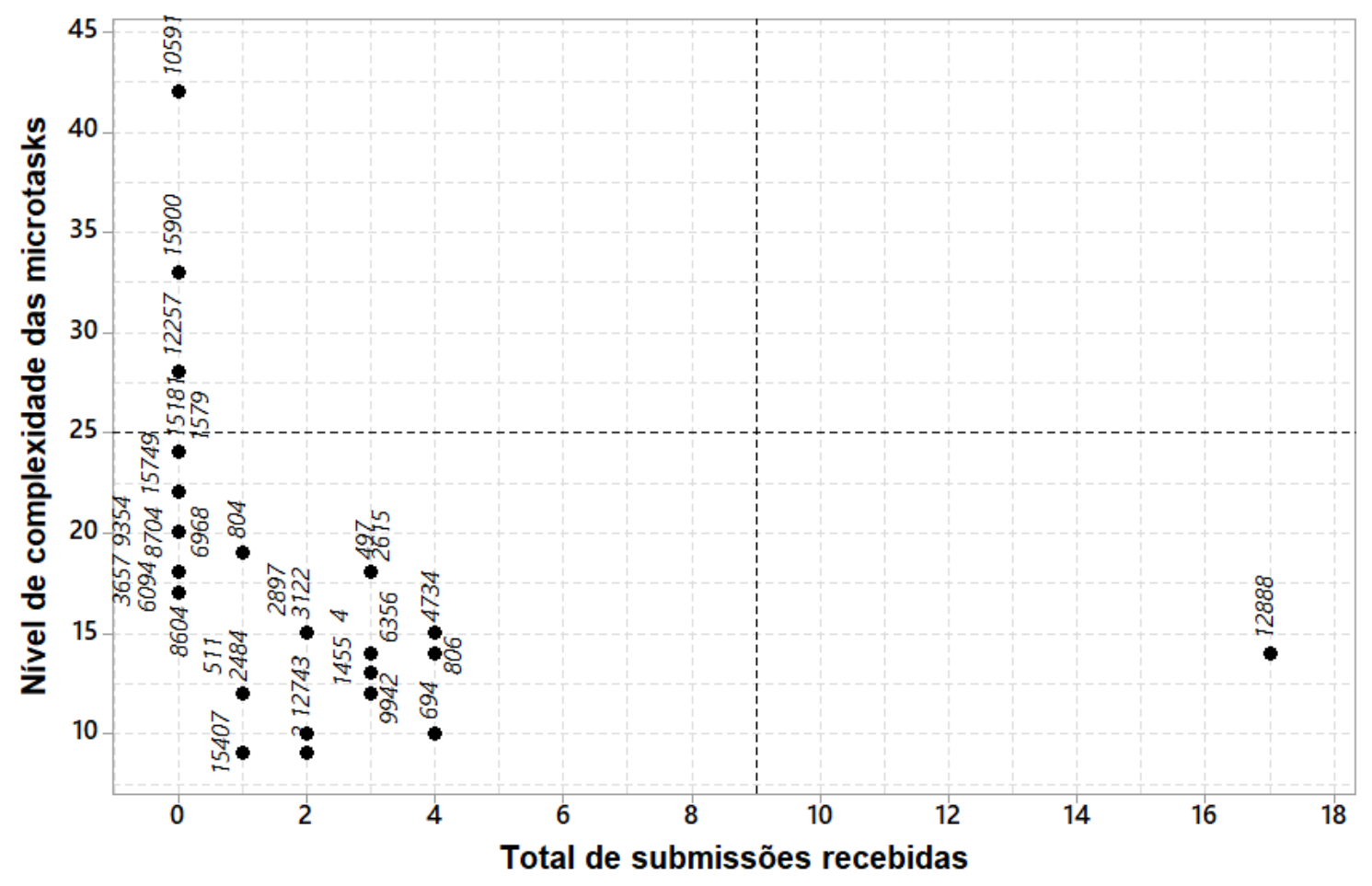

Figura 6. Dispersão entre complexidade e submissões das microtasks

A tendência natural das microtasks mais simples concentraram maior índice de submissão também foi satisfeita. Observou-se que tais tarefas, expostas nos quadrantes inferiores, alcançaram um índice entre 1 e 5 submissões. Destacando que a simplicidade, além de ter angariado maior registro também alcançou uma ou mais submissões. Isso é diametralmente oposto aos quadrantes superiores em que as três microtasks mais complexas não alcançaram nenhuma submissão.

\subsection{Discussão}

As QPs que nortearam este trabalho investigaram como a complexidade influenciou e afetou a finalização, o registro e a submissão em cada microtask analisada. $\mathrm{O}$ foco dessa análise foi avaliar se o método proposto seria eficiente em mensurar a complexidade e identificar a sua influência na execução de uma microtask.

Os resultados obtidos com a investigação da $\mathrm{QP}_{1}$ demonstraram que o método proposto havia classificado corretamente as microtasks em referência a sua complexidade e sua taxa de finalização. Essa percepção foi alcançada, principalmente, devido aos índices alcançados na amostra analisada. O que foi notado durante essa análise é que as microtasks classificadas como simples pelo método alcançaram quase $90 \%$ de conclusão, cenário contrário ao das microtasks complexas, que tiveram pouco mais de $13 \%$ de finalização.

Ainda tratando-se da análise da $\mathrm{QP}_{1}$ também é pertinente destacar que houve casos amostrais que não foram classificados corretamente. Duas microtasks simples não foram executadas e duas microtasks complexas lograram êxito em sua finalização. $\mathrm{O}$ aprofundamento desse tópico demonstrou que poderiam existir outras variáveis 
influenciando a própria complexidade da microtask, como o tipo da tarefa (teste, desenvolvimento ou design), além da falta de atenção aos requisitos obrigatórios. Apesar dessas constatações o método proposto foi capaz de constatar a complexidade corretamente nas outras 26 amostras analisadas, demostrando que o mesmo pode ser aperfeiçoado futuramente para tratar tais questões.

No caso da análise da $\mathrm{QP}_{2}$ notou-se como a complexidade de uma microtask também atuou no índice de registros e de submissões. E, novamente, o método foi eficiente ao classificar as microtasks como complexas ou simples e identificar essa influência. Os resultados exibiram que as tarefas mais simples receberem maior atenção dos crowd workers, e naturalmente, tiveram maiores índices de submissões. O que remete a sua taxa de sucesso de finalização. A respeito dessa questão ainda é pertinente destacar que as microtasks complexas podiam até atrair participantes, entretanto, o fluxo de submissão era extremamente baixo.

Nesse sentido ainda é pertinente destacar uma dinâmica do modelo de desenvolvimento de software CS e sua relação com a complexidade. Em uma ponta do modelo residem os crowdsourcers, que buscam solucionar seus desafios via microtasks. Já na outra extremidade os crowd workers desejam submeter soluções para angariar as premiações e as conquistas. Em ambos os casos, porém, o que se notou por meio da condução deste estudo é que todos podem alcançar melhores resultados através da simplificação das microtasks. No caso dos crowdsourcers, as microtasks simples aumentam as chances de êxito na finalização. Já os crowd wokers despertam a sua atenção e foco movidos pela facilidade de submissão que uma tarefa simples emprega. Tornando assim o modelo mais sustentável e eficiente no ponto de vista de todos os participantes, inclusive da própria plataforma de desenvolvimento, que pode atingir melhores índices de sucesso.

\subsection{Limitações}

A condução deste estudo possui limitações que podem impactar diretamente na validade das conclusões obtidas. A despeito disso é relevante discuti-las, e sob tais circunstâncias, foram sumarizados os seguintes aspectos de validade propostos por [Wohlin et al.2012]:

- Validade de construção: refletir se os resultados expostos nas QPs estão diretamente relacionados ao que foi observado. Nesse ponto é importante destacar que foi realizada uma investigação nos metadados das microtasks, descartando outras análises, como entrevistas ou questionários, que poderiam expandir a concepção sobre o motivo de duas microtasks consideradas simples terem sido canceladas e de duas microtasks consideradas complexas serem executadas com sucesso.

- Validade interna: analisar a possibilidade de fatores desconhecidos ou desconsiderados atuarem na amostra analisada. Nessa perspectiva pode-se destacar que não foi possível assumir que as microtasks analisadas tiveram o mesmo destaque durante a etapa de registro. Ou seja, a plataforma $\mathrm{X}$ pode ter realizado campanhas de marketing patrocinado por clientes, deixando determinadas microtasks com maior destaque de visualização (como banners, propagandas ou anúncios no site), atraindo assim maior atenção dos crowd workers e recebendo maior índice de registro. 
- Validade externa: verificar até que ponto os resultados obtidos podem ser generalizados. Sob essa perspectiva a principal limitação refere-se ao tamanho amostral que pode dificultar a generalização da eficácia do método para outros ambientes de desenvolvimento de software CS.

- Confiabilidade: identificar a dependência entre os dados obtidos e a análise efetuada. Naturalmente, nesse cenário surge uma limitação imposta pela plataforma. Assim, para replicações futuras deve-se obter uma coleção de microtasks com disposição similar dos metadados utilizados nesta pesquisa. Ademais, a disponibilidade da baseline é de responsabilidade da própria plataforma $\mathrm{X}$ e independe aos autores desta pesquisa.

\section{Conclusão e Trabalhos Futuros}

Este trabalho apresentou um método para determinar a complexidade de uma microtask. De modo resumido, foi gerado um cálculo de complexidade, avaliado por meio de um estudo de caso em uma plataforma de desenvolvimento de software CS.

Os resultados mostraram que o método aproximou-se de $90 \%$ de assertividade na amostra analisada, evidenciando que as microtasks mais simples tinham um índice de sucesso bem superior as microtasks com maior complexidade. Além disso, também foi descoberto que o aumento da dificuldade de uma microtask diminuía diametralmente o seu índice de registro e de submissão.

O maior impacto dos resultados obtidos nesta pesquisa contribuiu em dois aspectos: o primeiro, e já evidenciado neste estudo, é o fato de ainda não existirem - até o momento da escrita deste texto - abordagens, métodos ou cálculos eficientes e dedicados a determinar a complexidade de uma microtask em ambientes CS. Sendo assim, o método proposto neste estudo representa uma inovação para uma área da literatura ainda incipiente e desabastecida. O segundo aspecto reflete sobre a facilidade e portabilidade de aplicação do método proposto. Como foi demonstrando, o método foi capaz de medir a complexidade de microtasks de diferentes tipos, alcançado alta aderência em seu objetivo de determinar a complexidade independente da natureza de cada tarefa.

Em relação aos trabalhos futuros deseja-se automatizar o cálculo de complexidade por meio de uma aplicação online, além disso, existe o interesse na realização de novas pesquisas para amadurecer o método proposto, integrando soluções ao total de requisitos e tipos de microtasks.

\section{References}

Abhinav, K., Dubey, A., Jain, S., Bhatia, G. K., McCartin, B., and Bhardwaj, N. (2018). Crowdassistant: A virtual buddy for crowd worker. In 2018 IEEE/ACM 5th International Workshop on Crowd Sourcing in Software Engineering (CSI-SE), pages 17-20. doi: https://doi.org/10.1145/3195863.3195865 [GS Search]

Aipe, A. and Gadiraju, U. (2018). Similarhits: Revealing the role of task similarity in microtask crowdsourcing. HT. [GS Search] 
Chandra, G., Gupta, D. L., and Malviya, K. (2012). Some observations based on comparison of mood and ck software metrics suites for object oriented system. International Journal of Computer Science and Technology, 3(3).

de Deus, W. S., Barros, R. M., and L'Erario, A. (2016). Um modelo para o gerenciamento do crowdsourcing em projetos de software. In I Workshop sobre Aspectos Sociais, Humanos e Economicos de Software (WASHES'2016). [GS Search]

de Deus, W. S., Fabri, J. A., and L'Erario, A. (2017). The management of crowdsourcing software projects: A systematic mapping. In 2017 12th Iberian Conference on Information Systems and Technologies (CISTI), pages 1-7. doi: https://doi.org/10.23919/CISTI.2017.7975711 [GS Search]

de Freitas Junior, M., Fantinato, M., and Sun, V. (2015). Improvements to the function point analysis method: A systematic literature review. IEEE Transactions on Engineering Management, 62(4):495-506. doi: https://doi.org/10.1109/TEM.2015.2453354 [GS Search]

Dubey, A., Abhinav, K., Taneja, S., Virdi, G., Dwarakanath, A., Kass, A., and Kuriakose, M. S. (2016). Dynamics of software development crowdsourcing. In 2016 IEEE 11th International Conference on Global Software Engineering (ICGSE), pages 49-58. doi: https://doi.org/10.1109/ICGSE.2016.13 [GS Search]

Dwarakanath, A., Chintala, U., Shrikanth, N. C., Virdi, G., Kass, A., Chandran, A., Sengupta, S., and Paul, S. (2015). Crowd build: A methodology for enterprise software development using crowdsourcing. In 2015 IEEE/ACM 2nd International Workshop on CrowdSourcing in Software Engineering, pages 8-14. doi: https://doi.org/10.1109/CSI-SE.2015.9 [GS Search]

Goke, N. and Freitag, E. (2014). Microtask platforms a win/win/win situation. In . Colletive Inteligence. Greicius, T. (2018). Multi-planet system found through crowdsourcing. disponivel em: https://www.nasa.gov/feature/jpl/multi-planet-systemfound-throughcrowdsourcing. NASA.

Hosseini, M., Phalp, K., Taylor, J., and Ali, R. (2014). The four pillars of crowdsourcing: A reference model. In 2014 IEEE Eighth International Conference on Research Challenges in Information Science (RCIS), pages 1-12. doi: https://doi.org/10.1109/RCIS.2014.6861072 [GS Search]

Howe, J. (2006a). Crowdsourcing: A definition. https://crowdsourcing.typepad.com/cs/2006/06/crowdsourcing a.html, Janeiro, 2019.

Howe, J. (2006b). The rise of crowdsourcing. Wired magazine, 14(6):1-4. https://www.wired.com/2006/06/crowds/, Janeiro, 2019.

Jacques, J. T. (2018). Microtask Design: Value, Engagement, Context, and Complexity. $\mathrm{PhD}$ thesis, University of Cambridge. doi: http://dx.doi.org/10.17863/CAM.18777 [GS Search]

Karim, M. R., Yang, Y., Messinger, D., and Ruhe, G. (2018). Learn or earn? - intelligent task recommendation for competitive crowdsourced software development. doi: http://dx.doi.org/10.24251/HICSS.2018.700 [GS Search]

Kittur, A., Smus, B., Khamkar, S., and Kraut, R. E. (2011). Crowdforge: Crowdsourcing complex work. In Proceedings of the 24th Annual ACM Symposium on User Interface 
Software and Technology, UIST '11, pages 43-52, New York, NY, USA. ACM. doi: https://doi.org/10.1145/2047196.2047202 [GS Search]

Kurve, A., Miller, D. J., and Kesidis, G. (2015). Multicategory crowdsourcing accounting for variable task difficulty, worker skill, and worker intention. IEEE Transactions on Knowledge and Data Engineering, 27(3):794-809. doi: https://doi.org/10.1109/TKDE.2014.2327026 [GS Search]

LaToza, T. D., Towne, W. B., Adriano, C. M., and van der Hoek, A. (2014). Microtask programming: Building software with a crowd. In Proceedings of the 27th Annual ACM Symposium on User Interface Software and Technology, UIST '14, pages 4354, New York, NY, USA. ACM. doi: https://doi.org/10.1145/2642918.2647349 [GS Search]

LaToza, T. D. and v. d. Hoek, A. (2015). A vision of crowd development. In 2015 IEEE/ACM 37th IEEE International Conference on Software Engineering, volume 2, pages 563-566. doi: https://doi.org/10.1109/ICSE.2015.194

Mao, K., Capra, L., Harman, M., and Jia, Y. (2017). A survey of the use of crowdsourcing in software engineering. Journal of Systems and Software, 126:57 - 84.

Mao, K., Yang, Y., Li, M., and Harman, M. (2013). Pricing crowdsourcing-based software development tasks. In 2013 35th International Conference on Software Engineering (ICSE), pages 1205-1208. doi: https://doi.org/10.1016/j.jss.2016.09.015 [GS Search]

Naik, N. (2016). Crowdsourcing, open-sourcing, outsourcing and insourcing software development: A comparative analysis. In 2016 IEEE Symposium on Service-Oriented System Engineering (SOSE), pages 380-385. doi: https://doi.org/10.1109/SOSE.2016.68 [GS Search]

Nakatsu, R. T., Grossman, E. B., and Iacovou, C. L. (2014). A taxonomy of crowdsourcing based on task complexity. Journal of Information Science, 40(6):823834.

Sui, D., Elwood, S., and Goodchild, M. (2012). Crowdsourcing Geographic Knowledge: Volunteered Geographic Information (VGI) in Theory and Practice. Springer Publishing Company, Incorporated. doi: https://www.doi.org/10.1007/978-94-0074587-2

TopCoder (2017). Projects - topcoder crowdsourcing.

Tranquillini, S., Daniel, F., Kucherbaev, P., and Casati, F. (2015). Modeling, enacting, and integrating custom crowdsourcing processes. ACM Trans. Web, 9(2). Doi: https://doi.org/10.1145/2746353 [GS Search]

Wei Li, Michael N. Huhns, W.-T. T. W. W. (2015). Crowdsourcing Cloud-Based Software Development. Springer, first edition. doi: https://www.doi.org/10.1007/9783-662-47011-4

Winkler, D., Sabou, M., Petrovic, S., Carneiro, G., Kalinowski, M., and Biffl, S. (2017). Improving Model Inspection with Crowdsourcing. IEEE/ACM 4th International Workshop on Crowdsourcing in Software Engineering (CSI-SE), pages 30-40. doi: http://doi.ieeecomputersociety.org/10.1109/CSI-SE.2017.2 [GS Search] 
Wohlin, C., Runeson, P., Host, M., Ohlsson, M. C., Regnell, B., and Wesslen, A. (2012). Experimentation in software engineering. Springer Science \& Business Media. doi: https://www .doi.org/10.1007/978-3-642-29044-2

Xiao, L. and Paik, H. Y. (2014). Supporting complex work in crowdsourcing platforms: A view from service-oriented computing. In 2014 23rd Australian Software Engineering Conference, pages 11-14. doi: https://doi.org/10.1109/ASWEC.2014.11 [GS Search]

Yang J. Y. Redi, J. Demartini, G. B. A. (2016). Modeling task complexity in crowdsourcing. Fourth AAAI Conference on Human Computation and Crowdsourcing.

Yin, R. K. (2015). Estudo de Caso: Planejamento e Métodos. Bookman editora. 